\title{
Simulation of steady-state cuttings transport through a horizontal annulus channel
}

\author{
Yaroslav Ignatenko ${ }^{1, *}$, Andrey Gavrilov ${ }^{2}$, Oleg Bocharov ${ }^{1}$ and Roland May $^{3}$ \\ ${ }^{1}$ Baker Hughes Russia, Novosibirsk Technology Center, 630090 Novosibirsk, Russia \\ ${ }^{2}$ Institute of Thermophysics of SB RAS, Krasnoyarsk branch, 660036 Krasnoyarsk, Russia \\ ${ }^{3}$ Baker Hughes, a GE company, Celle Technology Center, 29221 Celle, Germany
}

\begin{abstract}
The current study is devoted to simulating cuttings transport by drilling fluid through a horizontal section of borehole with an annular cross section. Drill pipe rotates in fixed eccentric position. Steady-state flow is considered. Cuttings are rigid spheres with equal diameters. The carrying fluid is drilling mud with Herschel-Bulkley rheology. Suspension rheology depends on local shear rate and particles concentration. Continuous mixture model with algebraic equation for particles slipping velocity is used. Two hydrodynamic regimes are considered: axial flow without drill pipe rotation and with drill pipe rotation. In the case of axial flow was shown that increasing of power index $n$ and consistency factor $k$ increases pressure gradient and decreases cuttings concentration. Increasing of yield stress leads to increasing of pressure gradient and cuttings concentration. Cuttings concentration achieves constant value for high yield stress and not depends on it. Rotation of the drill pipe significantly changes the flow structure: pressure loss occurs and particles concentration decreases in the cross section. Two basic regimes of rotational flow are observed: domination of primary vortex around drill pipe and domination secondary vorticity structures. Transition between regimes leads to significant changes of flow integral parameters.
\end{abstract}

\section{Introduction}

One of the most important tasks for drilling fluid is to transport cuttings from the bottom of the well to the surface. In deviated and horizontal wellbores, this task can be complicated by cuttings particles sedimentation $[1,2]$. There, the solid particles build a cuttings bed, a stationary or slow-moving layer of locally high particle concentration. The optimization of the cuttings transport requires an understanding of the cuttings bed behavior mechanism. The height of the cuttings bed and the additional pressure drop due to the bed are of particular interest for the optimization engineer. The physical problem is described here as a steady-state transport phenomenon.

In the study of Ofei et al. [3, 4] influence of drill pipe rotation was numerically investigated. In article Mokhtari et al. [5] investigated influence of yield stress. In series of experimental studies dunes structures were studied $[6,7]$.

* Corresponding author: yaroslav.ignatenko@bakerhughes.com 


\section{Model description}

Steady-state flows in annuli are considered for laminar and turbulent flows. The HerschelBulkley rheological model describes the carrier fluid. Carrier fluid viscosity is $\mu=k \dot{\gamma}^{n-1}+\tau_{0} / \dot{\gamma}$, where $k$ is the consistency factor, $n$ is the power index, $\tau_{0}$ the yield stress, $\dot{\gamma}$ is the local shear rate. To describe the motion of the mixture, an inter-penetrating continual heterogeneous approach is used. Each phase has its own properties and state described by density, velocity, concentration, pressure, and viscosity. Mixture properties characterization is based on the summation of the individual phase properties. The twophase flow model is based on the mixture model with an algebraic equation for the relative velocity between the phases [8].

For the viscosity of the mixture, an empirical expression is used [9]. To predict turbulent characteristics of non-Newtonian fluid, the corrected two-parameter RANS model $(k-\omega \mathrm{SST})$ is used [10]. For normal stress in laminar flows with high particle concentration, we use the isotropic version of SBM (suspension balance model) [11]. For the nonNewtonian media, the drag force is found from correlations obtained by simulation of the motion of a single spherical particle in the unbounded fluid [12]. A correction factor is introduced that accounts for the influence of particles on the particle drag [13]. The lift force is calculated using Saffman's formula [14] with a correction factor [15] to apply it to high Reynolds numbers. The simplest formula for particle diffusion due to turbulent pulsations is based on the gradient hypothesis [8]. Current model and its testing more detailed described in paper [16].

\section{Problem statement}

We considered steady-state and homogenous along the channel particles (cuttings) transport by a Herschel-Bulkley fluid through an annular channel with an outer diameter $D_{o}$ and an inner diameter $D_{i}$. Flow rates of fluid and particles were considered as given values. The drill pipe (inner cylinder of annular channel) rotates with a given velocity (RPM), its position was set by the eccentricity $e=2 \Delta /\left(D_{o}-D_{i}\right)$, here $\Delta$ distance between center of cylinders. Inner cylinder is shifted downward. Non-slip boundary condition for mixture velocity and no particle diffusion or disperse phase concentration $\partial \alpha / \partial n=0$ were set on walls (inner and outer cylinders).

Table 1. Fluid and particles properties

\begin{tabular}{|l|l|}
\hline Parameter & Value, dimension \\
\hline$k$ & $0.1381 \mathrm{~Pa} \cdot \mathrm{s}^{\mathrm{n}}$ \\
\hline$n$ & 0.8 \\
\hline$\tau_{0}$ & $2.56 \mathrm{~Pa}$ \\
\hline fluid density $\rho_{f}$ & $1414 \mathrm{~kg} / \mathrm{m}^{3}$ \\
\hline particles density $\rho_{p}$ & $2650 \mathrm{~kg} / \mathrm{m}^{3}$ \\
\hline particles diameter $d_{p}$ & $3 \mathrm{~mm}$ \\
\hline
\end{tabular}

Table 2. Channel and basic drilling parameters

\begin{tabular}{|l|l|}
\hline Parameter & Value, dimension \\
\hline outer diameter $D_{o}$ & $0.254 \mathrm{~m}(10$ inches $)$ \\
\hline inner diameter $D_{i}$ & $0.127 \mathrm{~m}(5$ inches $)$ \\
\hline fluid flow rate $Q_{\text {mud }}$ & $\begin{array}{l}0.0367 \mathrm{~m}^{3} / \mathrm{s} \\
(22001 / \mathrm{min})\end{array}$ \\
\hline $\begin{array}{l}\text { particles flow rate } \\
Q_{p}\end{array}$ & $4.36 \cdot 10^{-4} \mathrm{~m}^{3} / \mathrm{s}$ \\
\hline inner cylinder $R P M$ & $\begin{array}{l}100 \text { revolutions per } \\
\text { minute }\end{array}$ \\
\hline
\end{tabular}


One of the typical horizontal drilling regimes was selected as a reference case. All parameters are described in Table 1 and Table 2. Flow characteristics were investigated by changing the drilling parameters and fluid rheology.

\section{Results and discussion}

The influence of the parameters on the cuttings bed erosion and the pressure drop in the annulus with cuttings was investigated by varying the values of the parameters defined in the reference case (Table 1 and Table 2). The viscosity of the fluid was changed by varying the power index $n$, yield stress $\tau_{0}$ and consistency factor $k$. String eccentricity and string rotation speed were also varied.

\subsection{Axial flow}





b

Fig. 1. Pressure gradient (a) and cuttings bed area (b) vs. power index $n$ for different eccentricity $e$ $(\mathrm{RPM}=0)$

Increased viscosity increased the friction on the channel's wall, but improved its carrying capabilities and reduced the cuttings bed area. This reaction can be seen when varying the power index $n$ for flows without drillstring rotation (Fig. 1). Here we call "cuttings concentration" a percent of annulus area filled by cuttings particles.

The same behavior was observed for the consistency factor $k$. It directly effects on fluid viscosity. Let us also mark that viscosity equal to consistency factor $k$ when the power index $n$ tends to one, and the yield stress $\tau_{0}$ - to zero.

An inverse dependence was observed while changing the yield stress $\tau_{0}$. The increase of this parameter $\tau_{0}$ increased the pressure drop (Fig. 2), while the cuttings bed area grew bigger (Fig. 2). Starting from $\tau_{0} \sim 5$, the Pa pressure gradient dependence on yield stress $\tau_{0}$ looks linear (Fig. 2a), and cuttings concentration standing constant (Fig. 2b). It means that the yield stress $\tau_{0}$ is strong enough to prevent washing away a cuttings bed. Yield stress significantly increases the apparent viscosity in the region of low shear rate, like the cuttings bed. This region is quasi-solid and increasing the yield stress increases this region. 


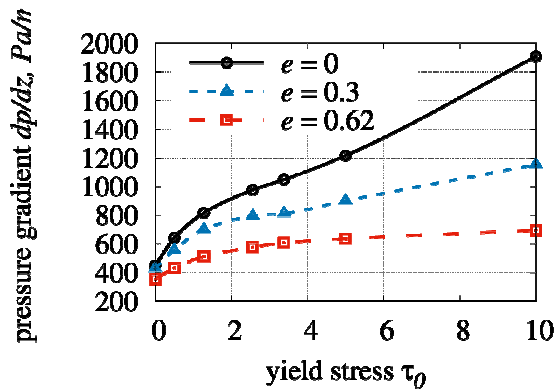

a

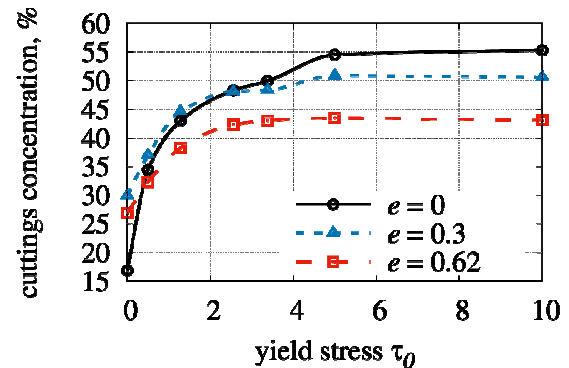

b

Fig. 2. Pressure gradient (a) and cuttings concentration (b) vs. eccentricity for different yield stress $\tau_{0}$ $(\mathrm{RPM}=0)$

\subsection{Spiral flow}

The rotation of the inner cylinder of annulus significantly changes flow structure, and step-wise changes of integral parameters occur. It can be explained by rearrangement of a flow structure. For a 2D problem of pure fluid flow in an annulus, it is also known that changes in such parameters as eccentricity, fluid rate and rheology cause transitional changes in the flow [17]. We observed two basic hydrodynamic regimes: domination primary rotational flow around drill pipe and domination of secondary reverse rotational flow. Transition between these two regimes can be caused by small changes of flow parameters like the eccentricity of annulus.

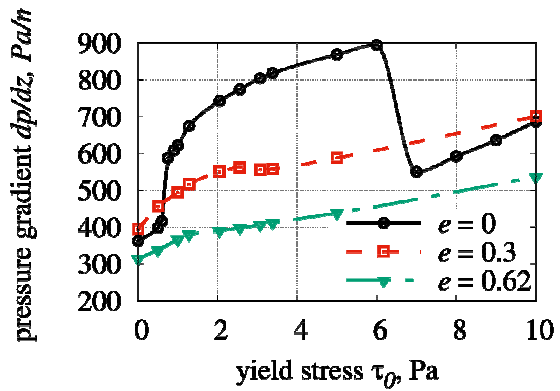

a

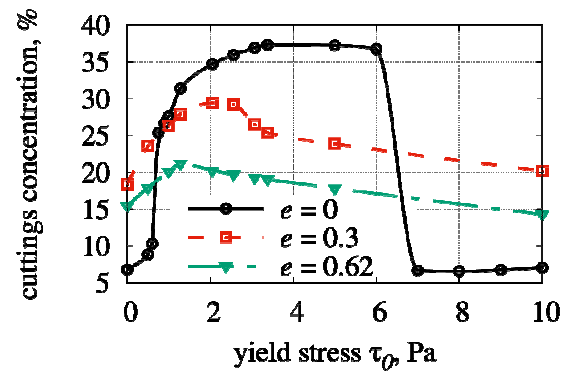

b

Fig. 3. Pressure gradient (a) and cuttings concentration area (b) vs. yield stresses $\tau_{0}$ and eccentricity $e$ $(\mathrm{RPM}=100)$

In the case of low or moderate yield stress values, a non-monotonic behavior of pressure drop on yield stress and eccentricity is observed (Fig. 3). In Fig. 3 for huge values $\tau_{0}>4$ we can see the same linear behavior of a pressure gradient on yield stresses $\tau_{0}$ as in the case without rotation in Fig. 2.

The behavior of the cuttings concentration in the cross section of the annular channel in this case largely coincides with the pressure for a small and moderate yield stress values (Fig. 3). However, for large values of yield stress a linear decrease of cuttings concentration exists, instead of a constant value as in the case without rotation (Fig. 2). 


\section{Conclusions}

Characteristics of steady state cuttings transport by Hershchel-Bulkley fluid through annular channel was investigated. In the case without drill pipe (inner cylinder) increasing of power index and consistency factor increases pressure gradient and decreases cuttings concentration. Increasing of yield stress increases both pressure gradient and cuttings concentration. Rotation of drill pipe (inner cylinder) cause appearance of vortex system. Domination of primary vortex flow with inner cylinder as a core or secondary reverse vortex flow significantly influence on cuttings transport characteristics. Flow rearrangement (changes of domination vortex) can cause of small changes of geometry or one of flow parameter.

The authors thank Baker Hughes, a GE Company for the permission to publish this article.

\section{References}

1. N.B. Egenti, proceedings of SPE Nigeria Annual International Conference and Exhibition (Society of Petroleum Engineers, 2014)

2. G. Jiimaa, Ph.D. thesis, U. of Stavanger, Faculty of Science and Technology (2013)

3. T.N. Ofei, S. Irawan, W. Pao, Journal of Petroleum Engineering 2014, 1 (2014)

4. T. Ofei, S. Alhemyari, proceedings of 015 International Field Exploration and Development Conference (IFEDC 2015)

5. M. Mokhtari, M. Ermila, A. Tutuncu, proceedings 46th U.S. Rock Mechanics/Geomechanics Symposium, 24-27 June, Chicago, Illinois (American Rock Mechanics Association, 2012)

6. R.W.S.R. Khatibi, Milad; Time, International Journal of Multiphase Flow (2017)

7. M.K.E. Rodriguez Corredor, Fabio Ernesto; Bizhani, Journal of Petroleum Science and Engineering 147 (2016)

8. M. Manninen, V. Taivassalo, S. Kallio, On the mixture model for multiphase flow (Julkaisija Utgivare, 1996)

9. $\quad$ N.S. Cheng, A.W.K. Law, Powder Technology 129, 156 (2003)

10. A.A. Gavrilov, V.Y. Rudyak, Journal of Non-Newtonian Fluid Mechanics 227, 45 (2016)

11. R.M. Miller, J.F. Morris, Journal of Non-Newtonian Fluid Mechanics 135, 149 (2006)

12. A.A. Gavrilov, K.A. Finnikov, E.V. Podryabinkin, Journal of Engineering Thermophysics 26, 197 (2017)

13. J. Richardson, W. Zaki, Chemical Engineering Research and Design 75, S82 (1997)

14. P.G. Saffman, Journal of Fluid Mechanics 31, 624 (1968)

15. J.B. McLaughlin, Journal of Fluid Mechanics 224, 261 (1991)

16. Ya. Ignatenko, A. Gavrilov, O. Bocharov, R. May, Proceedings of the OMAE2018, Spain, Madrid, June 17-22(2018)

17. E.V. Podryabinkin, V.Y. Rudyak, Journal of Engineering Thermophysics 23, 137 (2014) 